\section{Author Reply: Contralateral Prophylactic Mastectomy Overtreatment: Expectations from Personal Genomics for Tailored Breast Cancer Surgery}

\section{TO THE EDITORS:}

We appreciate the comments from Dr. Zografos and Dr. Roukos regarding our research on the use of contralateral prophylactic mastectomy (CPM). We previously reported that the CPM rate in the United States increased by more than $150 \%$ from 1998 to 2003 among patients with unilateral invasive breast cancer. ${ }^{1}$ In this study, these trends continued to the end of the study period with no plateau effect. We suspect that CPM rates have increased since 2003, but have not conducted such research.

The annual risk of contralateral breast cancer is low (about $0.6 \%$ ) for most patients. However, for patients with BRCA mutations, the annual risk is about $3 \%$ with a 10 year cumulative risk of about $30 \%$. Ideally, the decision to proceed with CPM should be guided by genetic risk assessment. In the current study, only $7.9 \%$ of patients who underwent CPM had preoperative BRCA testing.

The reasons for increased CPM rates are not clear. Perhaps, improved mastectomy and reconstruction techniques, increased awareness of genetic breast cancer, or changing attitudes regarding breast-conserving treatment all contribute to the observed trends.
Although absolute indications for CPM are not established, the Society of Surgical Oncology has published criteria that physicians should consider for mastectomy of the contralateral, intact breast; reasons for CPM may include diffuse microcalcifications, lobular carcinoma in situ, atypical hyperplasia, multicentric breast cancer, strong family history, the presence of a BRCA1 or BRCA2 mutation, dense breast tissue, or a large remaining breast (which complicates symmetric reconstructive techniques). ${ }^{2}$

Despite the availability of guidelines and genetic risk assessment, many patients choose bilateral mastectomy because of the fear of cancer occurring in the contralateral breast. Others choose bilateral mastectomy to avoid future surveillance or to achieve breast symmetry. Future research should focus on the development of decision aids for patients and surgeons.

Amanda Arrington, MD, and Todd Tuttle, MD, MS Department of Surgery, University of Minnesota, Minneapolis, MN

e-mail: tutt1006@umn.edu

Published Online: 24 November 2009

(C) Society of Surgical Oncology 2009

\section{REFERENCES}

1. Tuttle TM, Habermann EB, Grund EH, Morris, TJ, Virnig, BA. Increasing use of contralateral prophylactic mastectomy for breast cancer patients: a trend toward more aggressive surgical treatment. J Clin Oncol. 2007;25:5203-9.

2. Statement on prophylactic mastectomy. http://www.surgonc.org. 